\title{
ISMuLT skeletal muscles injuries Guidelines
}

\section{Nicola Maffulli ${ }^{1}$ \\ Gianni Nanni²}

1 Head of Department of Physical and Rehabilitation Medicine, University of Salerno, Azienda Ospedaliera San Giovanni Di Dio e Ruggi d'Aragona, Salerno, Italy

Centre for Sports and Exercise Medicine, Queen Mary University of London, Barts and The London School of Medicine and Dentistry, Mile End Hospital, London, UK

2 Isokinetic Medical Group, Head of the Medical Staff of Bologna Football Club 1909, Bologna, Italy

\section{Corresponding author:}

Nicola Maffulli

Centre for sport and Exercise Medicine,

Queen Mary University of London,

Barts and The London School of Medicine and Dentistry, Mile End Hospital, UK

Department of Physical and Rehabilitation Medicine, University of Salerno, Italy

E-mail: n.maffulli@qmul.ac.uk

It is always difficult to produce guidelines. Indeed, when, during my clinical career, I have been faced with such tools, I cringed, and my heart sank.

ISMuLT prides itself to be at the forefront in research and clinical aspects in the science and art of manag- ing muscle, ligament and tendon ailments. In 2012, our membership asked for guidance regarding muscle injuries. With some trepidation, in that year the Scientific Committee decided that the call had to be answered. Under the wise general supervision of Dr. Gianni Nanni, a widely read and superb sports physician, a varied group of health care professional started to work to analyse the literature, and ascertain whether the ivory tower of academia could be distilled into practical advice for on-the-field clinical practitioner.

I am proud to say that, after uncountable emails and teleconferences, and three face-to-face meetings, the results are here to see.

To Dr. Nanni my personal thanks, which are to be extended to all the individuals who took part in this: the whole ISMuLT is grateful to you. Also, many thanks to the various hosts who took charge to make sure that the working party was looked after appropriately during the various face-to-face meetings.

ISMULT has produced a version in English, which is published in the present issue of Muscles, Ligaments and Tendons Journal. A more extensive version will be published in Italian, and our readers will be able to access both.

A word of restraint. Guidelines are not meant to substitute expert clinical practice. As they are based on scientific evidence and on personal opinions, guidelines should be interpreted with a pinch (and at times more than one) of salt. Also, they need to be updated on a regular basis.

All the best 